\title{
Synthesis of a tetrahydroimidazo[2',1':2,3]thiazolo[5,4-c]pyridine derivative with Met inhibitory activity
}

\author{
Mercedes Amat, Andrea Kövér, Danica Jokic, Oscar Lozano, Maria Pérez, Nicola \\ Landoni, Fabiana Subrizi, Jesús Bautista, and Joan Bosch* \\ Laboratory of Organic Chemistry, Faculty of Pharmacy, and Institute of Biomedicine \\ (IBUB), University of Barcelona, 08028 Barcelona, Spain \\ E-mail:joanbosch@ub.edu
}

Dedicated to Prof. Benito Alcaide on the occasion of his $60^{\text {th }}$ birthday

\begin{abstract}
A straightforward synthesis of the Met antagonist JLK1360 involving an alkylationcyclocondensation process using aminothiazole $\mathbf{1}$ and nitrophenacyl bromide $\mathbf{2}$, reduction of the nitro group, and coupling of the resulting tetracyclic aniline $\mathbf{5}$ with an appropriate $\mathrm{N}$-acyl alanine derivative, is reported.
\end{abstract}

Keywords: 2-Aminothiazole, Met inhibitory activity, tyrosine kinase Met receptor, 2aryltetrahydro[2',1':2,3]thiazolo[5,4-c]pyridine

\section{Introduction}

The hepatocyte growth factor receptor, also known as the mesenchymal-epithelial transition factor (c-Met), is a tyrosine kinase receptor present in both normal and malignant cells. Its activation promotes a variety of effects, such as cell proliferation or angiogenesis, leading to invasiveness and metastasis. Moreover, it is well recognized that this receptor type is overexpressed and mutated in a variety of human cancer types and constitutes a therapeutic target for the development of selective new anti-cancer drugs. The main pharmacological interest is to find drugs capable of preventing receptor autophosphorylation.

In this context, compound JLK1360, in a concentration range between 100 to $250 \mathrm{nM}$, inhibited cell scattering and branching morphogenesis. ${ }^{1}$ These effects are due to its capacity to impair receptor signalling and restoration after degradation. From computational studies (docking and molecular dynamics) it is well assumed that this compound interacts with this receptor at the ATP-binding pocket. The high degree of selectivity of JLK1360 was assessed by computational and also biochemical studies. 


\section{Results and Discussion}

We report herein the details of the synthesis of JLK1360. The commercially available 2-amino5-benzyl-4,5,6,7-tetrahydrothiazolo[5,4-c]pyridine $\mathbf{1}^{2}$ was chosen as the starting material of the synthesis. We planned to take advantage of the 2-aminothiazole moiety of $\mathbf{1}$ to assemble the tricyclic tetrahydroimidazo[2',1':2,3]-thiazolo[5,4-c]pyridine skeleton by an alkylationcyclocondensation process with an appropriate phenacyl bromide. ${ }^{3}$ A final acylation of the C-2 $p$-aminophenyl substituent using an activated $N$-(phenylacetyl)alanine would complete the synthesis.

The key aniline tricyclic intermediate 5 was prepared in $40 \%$ overall yield in a straightforward manner by reaction of thiazolopyridine $\mathbf{1}$ with $p$-nitrophenacyl bromide $\mathbf{2}$ in refluxing $i$-PrOH, followed by reduction of the nitro moiety of the resulting 2-aryl substituted imidazothiazolopyridine 4 using $\mathrm{SnCl}_{2}$ in concentrated hydrochloric acid and methanol at reflux. Minor amounts of the chlorinated derivative $\mathbf{6}$ were also formed (Scheme 1). When the initial step was performed in refluxing toluene, alkylation at the ring nitrogen occurred to give the imino derivative $3,{ }^{4}$ which was subsequently cyclized to 4 , although in low overall yield ( 10\%), by heating in a methanol solution.

Significant ${ }^{1} \mathrm{H}$ NMR signals of compound $\mathbf{4}$ are two doublets due to the aromatic protons of the $p$-nitrophenyl substituent, a singlet for the proton of the imidazole moiety, two signals attributable to the $\mathrm{N}$-benzyl and $\mathrm{N}$-allyl methylene protons, and two triplets due to the $\mathrm{CH}_{2}-\mathrm{CH}_{2}$ fragment of the tetrahydropyridine ring.
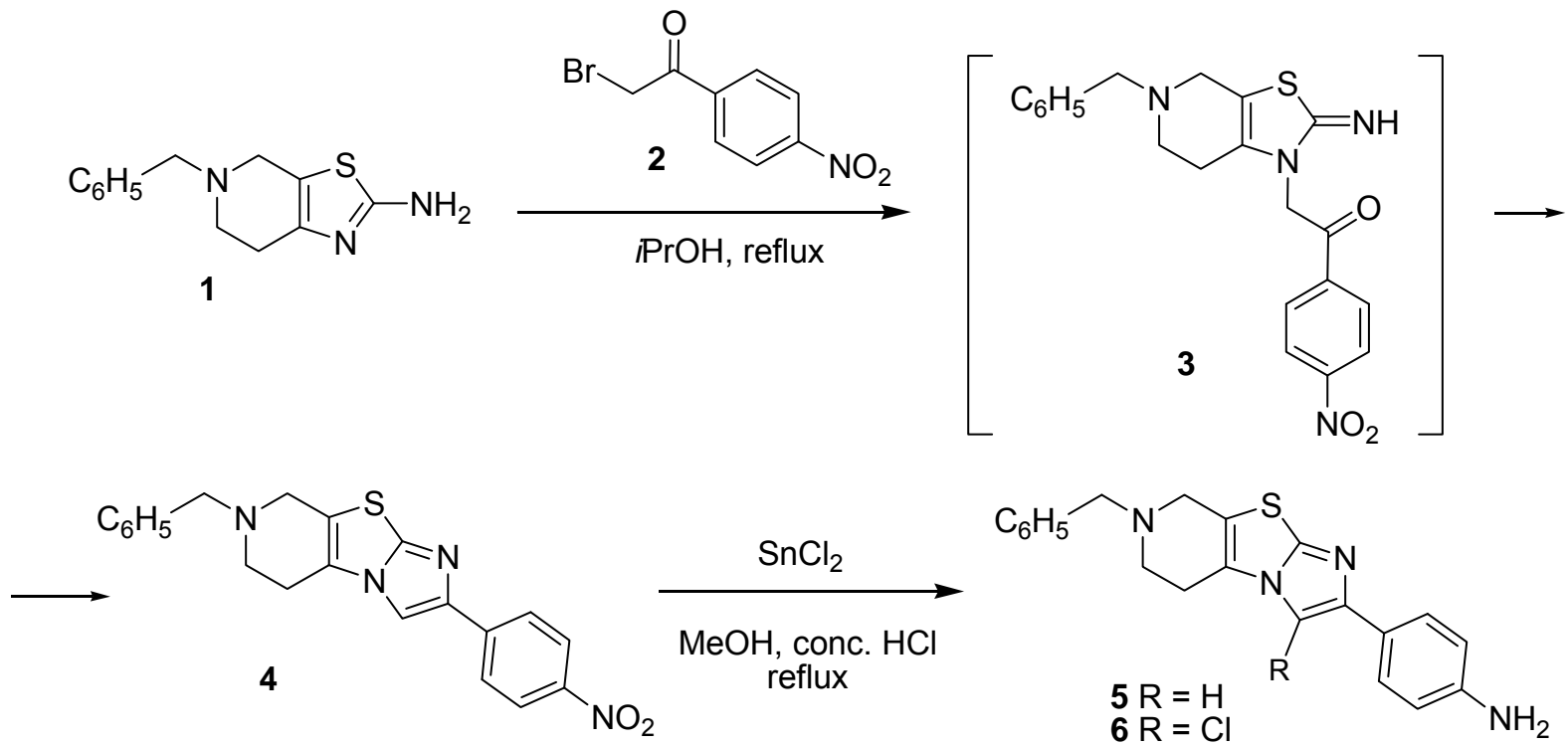

Scheme 1. Synthesis of the 2-aryltetrahydro[2',1':2,3]thiazolo[5,4-c]pyridine fragment. 
On the other hand, the required amido acid $\mathbf{8}$ was prepared by coupling of $(S)$-alanine methyl ester with 3,5-difluorophenylacetic acid using DCC as the condensing agent, followed by alkaline hydrolysis of the resulting amido ester $\mathbf{7}$, as outlined in Scheme $2 .^{5}$

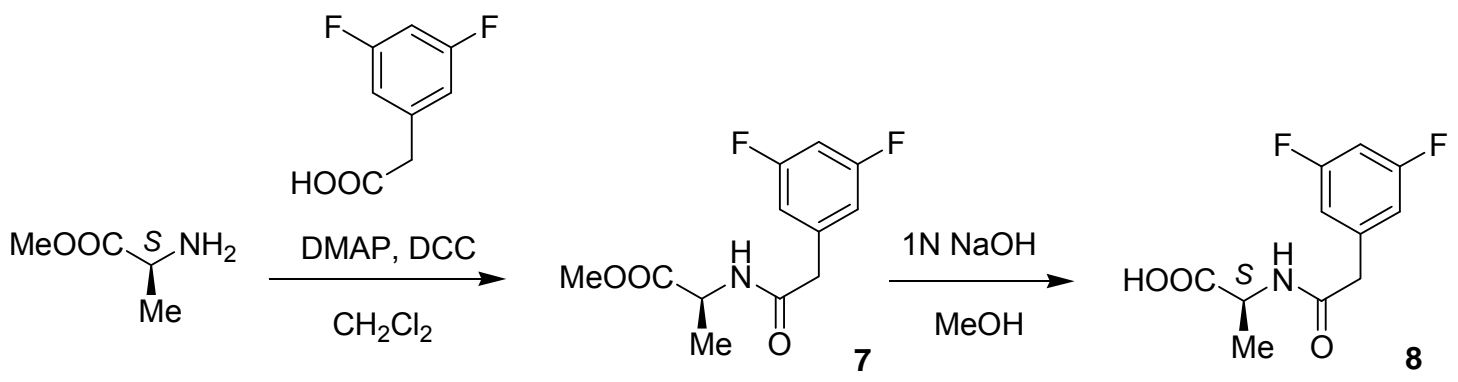

Scheme 2. Synthesis of amido acid $\mathbf{8}$.

The final step of the synthesis was the condensation reaction between the tricyclic aniline derivative 5 and $N$-acylalanine $\mathbf{8}$. We initially used $\mathrm{BOP}^{6}$ as the coupling reagent ${ }^{7}$ in the presence of HOBt and DIPEA (Scheme 3). Although the desired target JLK1360 was obtained in acceptable yield (50\%), its complete purification by column chromatography proved to be difficult due to the presence of a minor impurity. The use of $\mathrm{BOPCl}^{8}$ as the coupling reagent in the presence of HOBt and DIPEA gave more satisfactory results in terms of chromatographic purification, leading to pure JLK1360 in 47\% yield.

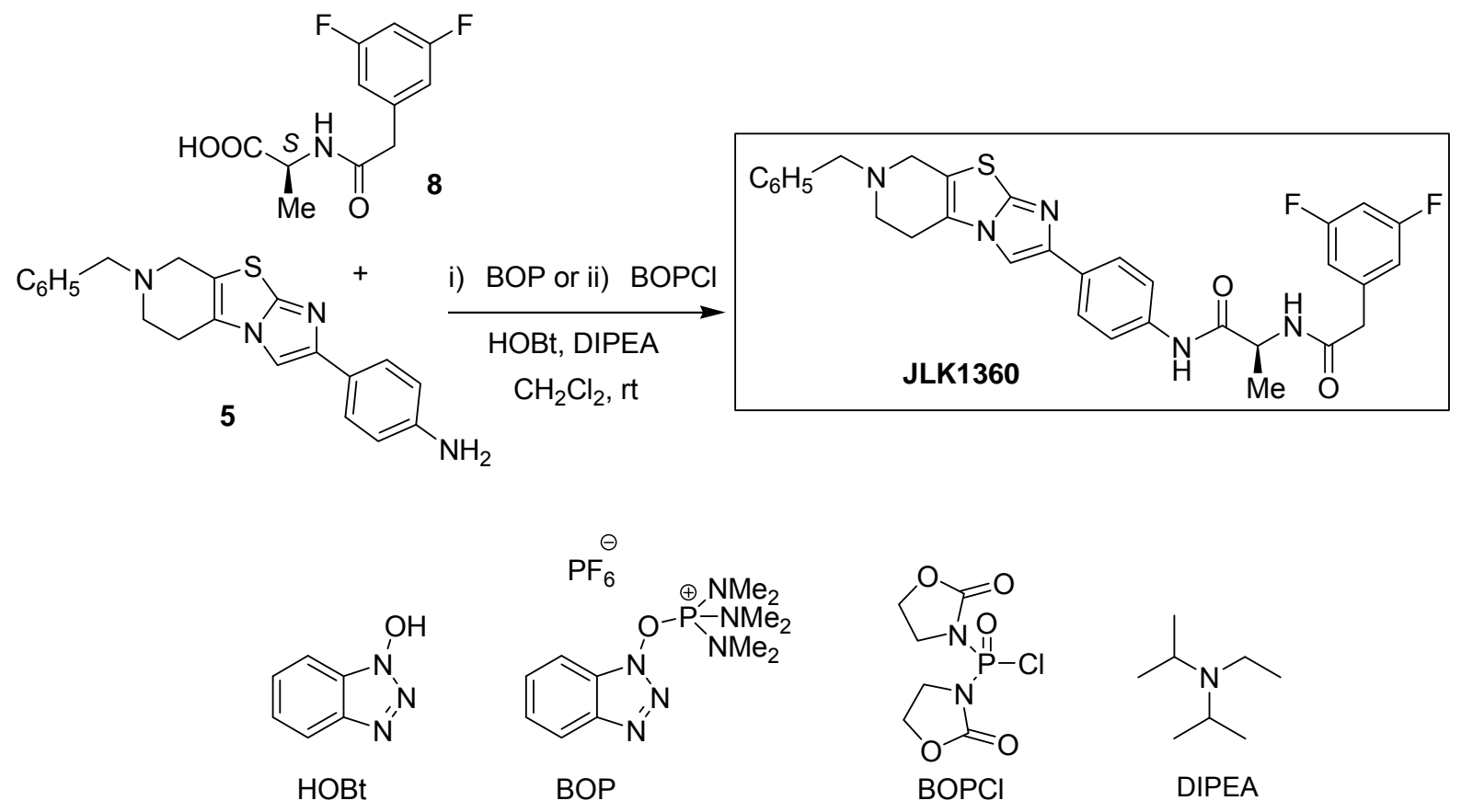

Scheme 3. Condensation reaction. 
Both the ${ }^{1} \mathrm{H}$ and ${ }^{13} \mathrm{C}$ NMR data were in complete agreement with the structure of JLK1360. Seven groups of signals are present in the aliphatic region of the ${ }^{1} \mathrm{H}$ NMR spectrum, attributable to the five methylene groups and the methine and methyl protons of the alanine moiety. The assignments (see Experimental Part) were confirmed by bidimentional COSY and HSQC experiments.

The use of aniline 5 as a platform for the synthesis of JLK1360 analogues following a similar synthetic sequence is in progress.

\section{Experimental Section}

General Procedures. Reagents and chemicals were purchased from commercial sources [Sigma-Aldrich S.A., Riedel-de Haën $\left(\mathrm{SnCl}_{2}\right)$, and Fluorochem (3,5-difluorophenylacetic acid)] and used as received. 2-Amino-5-benzyl-4,5,6,7-tetrahydrothiazolo[5,4-c]pyridine $\mathbf{1}$ was purchased from UKR Org. Synthesis Ltd. All non-aqueous reactions were performed under an argon or nitrogen atmosphere with dry, freshly distilled solvents using standard procedures. Drying of organic extracts during the work-up of reactions was performed over anhydrous $\mathrm{Na}_{2} \mathrm{SO}_{4}$ or $\mathrm{MgSO}_{4}$. Evaporation of solvents was accomplished with a rotatory evaporator. Thinlayer chromatography was done on $\mathrm{SiO}_{2}$ (silica gel $60 \mathrm{~F}_{254}$ ), and the spots were located by UV and either a $1 \% \mathrm{KMnO}_{4}$ solution or iodine. Chromatography refers to flash column chromatography and was carried out on $\mathrm{SiO}_{2}$ (silica gel 60, 230-400 mesh). NMR spectra were recorded at 300 or $400 \mathrm{MHz}\left({ }^{1} \mathrm{H}\right)$ and 75.4 or $100.6 \mathrm{MHz}\left({ }^{13} \mathrm{C}\right)$, and chemical shifts are reported in $\delta$ values downfield from TMS or relative to residual chloroform (7.26 ppm, $77.0 \mathrm{ppm})$ as an internal standard. Melting points were determined in a capillary tube and are uncorrected. Optical rotation was recorded on a Perkin Elmer Model 241 Polarimeter.

7-Benzyl-2-(p-nitrophenyl)-5,6,7,8-tetrahydroimidazo[2',1':2,3]thiazolo[5,4-c]pyridine (4). 2-Amino-5-benzyl-4,5,6,7-tetrahydrothiazolo[5,4-c]pyridine (1; 1.0 g, 4.08 mmol) and 2-bromo$p$-nitroacetophenone (2; $1.09 \mathrm{~g}, 4.48 \mathrm{mmol})$ were dissolved in anhydrous $i$-PrOH (6 mL), and the mixture was heated at reflux for 50 minutes. The reaction was controlled by TLC analysis (1:1 EtOAc-hexane). When the reaction was complete, the resulting mixture was cooled to $\mathrm{rt}$ and concentrated under vacuum to afford crude compound $4\left(\mathrm{R}_{f}=0.21\right.$ in 1:1 EtOAc-hexane), which was used in the next step without further purification. Column chromatography (1:1 EtOAchexane containing a few drops of $\mathrm{NEt}_{3}$ ) afforded pure 4 as an orange solid: $\mathrm{mp} 68-69{ }^{\circ} \mathrm{C}$; ${ }^{1} \mathrm{H}$ NMR (400 MHz, CDCl $\left.{ }_{3}\right) \delta 2.81(\mathrm{t}, J=6.0 \mathrm{~Hz}, 2 \mathrm{H}), 3.01$ (t, $\left.J=6.0 \mathrm{~Hz}, 2 \mathrm{H}\right), 3.61(\mathrm{~s}, 2 \mathrm{H}), 3.78$ (s, 2H), 7.27-7.37 (m, 5H, $\left.\mathrm{H}_{\mathrm{Ar}}\right), 7.70(\mathrm{~s}, 1 \mathrm{H}), 7.94\left(\mathrm{dm}, J=8.8 \mathrm{~Hz}, 2 \mathrm{H}, \mathrm{H}_{\mathrm{Ar}}\right), 8.22(\mathrm{dm}, J=8.8$ $\left.\mathrm{Hz}, 2 \mathrm{H}, \mathrm{H}_{\mathrm{Ar}}\right) ;{ }^{13} \mathrm{C}$ NMR (100.6 MHz, $\left.\mathrm{CDCl}_{3}\right) \delta 23.0\left(\mathrm{CH}_{2}\right), 48.7\left(\mathrm{CH}_{2}\right), 50.5\left(\mathrm{CH}_{2}\right), 61.4\left(\mathrm{CH}_{2}\right)$, $107.8(\mathrm{CH}), 120.6\left(\mathrm{C}_{\mathrm{Ar}}\right), 124.2\left(2 \mathrm{CH}_{\mathrm{Ar}}\right), 124.5\left(\mathrm{C}_{\mathrm{Ar}}\right), 125.2\left(2 \mathrm{CH}_{\mathrm{Ar}}\right), 127.6\left(\mathrm{CH}_{\mathrm{Ar}}\right), 128.5$ $\left(2 \mathrm{CH}_{\mathrm{Ar}}\right), 129.0\left(2 \mathrm{CH}_{\mathrm{Ar}}\right), 137.5,140.7,144.4,146.5\left(\mathrm{C}_{\mathrm{Ar}}\right) . \mathrm{MS}(\mathrm{EI}) \mathrm{m} / z$ (rel intensity) $391(\mathrm{M}+$, 
20), 384 (24), 369 (36), 368 (46), 367 (49), 366 (39), 351 (31), 350 (25), 337 (15), 277 (24), 276 (17), 261 (20), 261 (100), 260 (49), 259 (43), 258 (23), 257 (20) 122 (28), 106 (26).

2-(p-Aminophenyl)-7-benzyl-5,6,7,8-tetrahydroimidazo[2',1':2,3]thiazolo[5,4-c]pyridine (5). $\mathrm{SnCl}_{2}$ (2.53 g, $13.3 \mathrm{mmol}$ ) was added to a stirred suspension of the above crude 4 in $\mathrm{MeOH}$ $(90 \mathrm{~mL})$. Then, $37 \%$ aqueous $\mathrm{HCl}(90 \mathrm{~mL})$ was slowly added, and the resulting mixture was heated at reflux for 30 minutes. The reaction was controlled by TLC analysis (2:1 EtOAchexane). When the reaction was complete, $2 \mathrm{M}$ aqueous $\mathrm{K}_{2} \mathrm{CO}_{3}$ was added to the mixture until $\mathrm{pH}$ 9. The resulting precipitate was filtered and chromatographed (EtOAc containing a few drops of $\mathrm{NEt}_{3}$ ) to give compound 5 (588 mg, 40\% overall yield from $\mathbf{1}$; $\mathrm{R}_{f}=0.14$ in 2:1 EtOAchexane) as a brown solid. Minor amounts of the chlorinated derivative 6 (16 mg) were also isolated. 5: mp 135-137 ${ }^{\circ} \mathrm{C}$; ${ }^{1} \mathrm{H}$ NMR $\left(400 \mathrm{MHz} \mathrm{CDCl}_{3}\right) \delta 2.71(\mathrm{t}, J=6.0 \mathrm{~Hz}, 2 \mathrm{H}), 2.95(\mathrm{t}, J=$ $6.0 \mathrm{~Hz}, 2 \mathrm{H}), 3.56$ (s, 2H), 3.73 (s, 2H), $6.69\left(\mathrm{dm}, J=8.4 \mathrm{~Hz}, 2 \mathrm{H}, \mathrm{CH}_{\mathrm{Ar}}\right.$ ), 7.25-7.36 (m, 5H, $\left.\mathrm{CH}_{\mathrm{Ar}}\right), 7.39(\mathrm{~s}, 1 \mathrm{H}), 7.60\left(\mathrm{dm}, J=8.4 \mathrm{~Hz}, 2 \mathrm{H}, \mathrm{CH}_{\mathrm{Ar}}\right) ;{ }^{13} \mathrm{C} \mathrm{NMR}\left(100.6 \mathrm{MHz}, \mathrm{CDCl}_{3}\right) \delta 22.9$ $\left(\mathrm{CH}_{2}\right), 48.7\left(\mathrm{CH}_{2}\right)$; $50.8\left(\mathrm{CH}_{2}\right), 61.2\left(\mathrm{CH}_{2}\right), 104.1(\mathrm{CH}), 115.2\left(2 \mathrm{CH}_{\mathrm{Ar}}\right), 118.4,124.5,125.0$ $\left(\mathrm{C}_{\mathrm{Ar}}\right), 126.1\left(2 \mathrm{CH}_{\mathrm{Ar}}\right), 127.4\left(\mathrm{CH}_{\mathrm{Ar}}\right), 128.4\left(2 \mathrm{CH}_{\mathrm{Ar}}\right), 129.0\left(2 \mathrm{CH}_{\mathrm{Ar}}\right), 137.6,145.6,147.0,148.0$ $\left(\mathrm{C}_{\mathrm{Ar}}\right)$. HMRS calcd for $\left[\mathrm{C}_{21} \mathrm{H}_{20} \mathrm{~N}_{4} \mathrm{~S}+\mathrm{H}\right]$ : 361.1481, found: 361.1479. 6: mp 148-150 ${ }^{\circ} \mathrm{C} ;{ }^{1} \mathrm{H}$ NMR (400 MHz, $\left.\mathrm{CDCl}_{3}\right) \delta 2.94(\mathrm{t}, J=6.0 \mathrm{~Hz}, 2 \mathrm{H}), 3.13(\mathrm{~m}, 2 \mathrm{H}), 3.56$ (t, $\left.J=2.0 \mathrm{~Hz}, 2 \mathrm{H}\right), 3.74$ (s, 2H), $6.72\left(\mathrm{dm}, J=9.2 \mathrm{~Hz}, 2 \mathrm{H}, \mathrm{CH}_{\mathrm{Ar}}\right), 7.28-7.36\left(\mathrm{~m}, 5 \mathrm{H}, \mathrm{CH}_{\mathrm{Ar}}\right), 7.75(\mathrm{dm}, J=9.2 \mathrm{~Hz}, 2 \mathrm{H}$, $\left.\mathrm{CH}_{\mathrm{Ar}}\right) ;{ }^{13} \mathrm{C}$ NMR (100.6 MHz, $\left.\mathrm{CDCl}_{3}\right) \delta 23.7\left(\mathrm{CH}_{2}\right), 48.9\left(\mathrm{CH}_{2}\right), 50.6\left(\mathrm{CH}_{2}\right), 61.3\left(\mathrm{CH}_{2}\right), 105.1$ $\left(\mathrm{C}_{\mathrm{Ar}}\right), 114.9\left(2 \mathrm{CH}_{\mathrm{Ar}}\right), 119.3,123.4,125.4\left(\mathrm{C}_{\mathrm{Ar}}\right), 127.5\left(\mathrm{CH}_{\mathrm{Ar}}\right), 127.8\left(2 \mathrm{CH}_{\mathrm{Ar}}\right), 128.5\left(2 \mathrm{CH}_{\mathrm{Ar}}\right)$, $129.0\left(2 \mathrm{CH}_{\mathrm{Ar}}\right), 137.5,140.7,145.9,146.4\left(\mathrm{C}_{\mathrm{Ar}}\right)$. HMRS calcd for $\left[\mathrm{C}_{21} \mathrm{H}_{19} \mathrm{ClN}_{4} \mathrm{~S}+\mathrm{H}\right]$ : 395.1092, found: 395.1086.

(S)-Methyl 2-[(3,5-difluorophenyl)acetamido]propanoate (7). DCC (8.66 g, $42.0 \mathrm{mmol}$ ) was added to a solution of L-alanine methyl ester hydrochloride (4.0 g, $28.0 \mathrm{mmol})$ and 3,5difluorophenylacetic acid $(4.8 \mathrm{~g}, 28.0 \mathrm{mmol})$ in $\mathrm{CH}_{2} \mathrm{Cl}_{2}(320 \mathrm{~mL})$. After stirring at rt for 10 minutes, DMAP (1.71 g, $14.0 \mathrm{mmol}$ ) was added in small portions, and the resulting mixture was stirred for $48 \mathrm{~h}$ and then concentrated under vacuum. Flash chromatography (1:1 EtOAc-hexane) afforded compound 7 (5.90 g, 82\% yield; $\mathrm{R}_{f}=0.54$ in 1:1 EtOAc-hexane) as a pale yellow solid: mp 75-76 ${ }^{\circ} \mathrm{C}$; ${ }^{1} \mathrm{H}$ NMR (400 MHz, $\mathrm{CDCl}_{3}$ ) $\delta 1.39$ (d, $J=7.2 \mathrm{~Hz}, 3 \mathrm{H}, \mathrm{CH}_{3}$ ), 3.55 (s, 2H), 3.74 (s, $3 \mathrm{H}, \mathrm{OCH}_{3}$ ), 4.58 (qd, $J=7.2 \mathrm{~Hz}, 1 \mathrm{H}$ ), 6.26 (br s., $1 \mathrm{H}, \mathrm{NH}$ ), 6.73 (tt, $J=9.2,2.4 \mathrm{~Hz}, 1 \mathrm{H}, \mathrm{CH}_{\mathrm{Ar}}$ ), $6.84\left(\mathrm{~m}, 2 \mathrm{H}, \mathrm{CH}_{\mathrm{Ar}}\right) ;{ }^{13} \mathrm{C} \mathrm{NMR}\left(100.6 \mathrm{MHz}, \mathrm{CDCl}_{3}\right) \delta 18.2\left(\mathrm{CH}_{3}\right), 42.8\left(\mathrm{CH}_{2}\right), 48.2(\mathrm{CH}), 52.5$ $\left(\mathrm{CH}_{3}\right), 102.8\left(\mathrm{t}, J_{\mathrm{C}-\mathrm{F}}=24.9 \mathrm{~Hz}, \mathrm{CH}_{\mathrm{Ar}}\right), 112.2\left(\mathrm{dd}, J_{\mathrm{C}-\mathrm{F}}=18.7,7.0 \mathrm{~Hz}, \mathrm{CH}_{\mathrm{Ar}}\right), 138.2\left(\mathrm{t}, J_{\mathrm{C}-\mathrm{F}}=10.1\right.$ $\mathrm{Hz}, \mathrm{C}_{\mathrm{Ar}}$ ), $163.0\left(\mathrm{dd}, J_{\mathrm{C}-\mathrm{F}}=248.5,12.5 \mathrm{~Hz}, \mathrm{C}_{\mathrm{Ar}}\right.$ ), 169.0, $173.3(\mathrm{C}=\mathrm{O})$. HMRS calcd for $\left[\mathrm{C}_{12} \mathrm{H}_{13} \mathrm{~F}_{2} \mathrm{NO}_{3}+\mathrm{H}\right]:$ 258.0936, found: 258.0936.

(S)-2-[(3,5-Difluorophenyl)acetamido]propanoic acid (8). $1 \mathrm{M}$ aqueous $\mathrm{NaOH}$ (79 mL, 78.8 mmol) was added to a solution of ester 7 (4.5 g, $17.5 \mathrm{mmol}$ ) in $\mathrm{MeOH}$ (196 mL), and the mixture was stirred at rt for $24 \mathrm{~h}$. Water was added, and the $\mathrm{MeOH}$ was eliminated under vacuum. The aqueous layer was acidified to $\mathrm{pH} 3$, and the solution was extracted with EtOAc. The combined extracts were washed with water and brine, dried, and concentrated. Saturated aqueous $\mathrm{NaHCO}_{3}$ was added to the resulting solid residue, and the suspension was extracted with $\mathrm{CH}_{2} \mathrm{Cl}_{2}$. The 
aqueous phase was neutralized and extracted with EtOAc. The combined ethyl acetate extracts were washed with water and brine, dried, and concentrated under vacuum to afford pure compound 8 (2.89 g, 68\% yield; $\mathrm{R}_{f}=0.15$ in EtOAc containing a few drops of $\mathrm{NEt}_{3}$ ) as a white solid: mp 108-109 ${ }^{\circ} \mathrm{C} ;{ }^{1} \mathrm{H}$ NMR (300 MHz, CD $\left.\mathrm{OD}\right) \delta 1.39\left(\mathrm{~d}, J=7.2 \mathrm{~Hz}, 3 \mathrm{H}, \mathrm{CH}_{3}\right), 3.54(\mathrm{~d}, J=$ $14.8 \mathrm{~Hz}, 1 \mathrm{H}, \mathrm{CH}_{2}$ ), 3.58 (d, $J=14.8 \mathrm{~Hz}, 1 \mathrm{H}, \mathrm{CH}_{2}$ ), 4.37 (q, $\left.J=7.2 \mathrm{~Hz}, 1 \mathrm{H}, \mathrm{CH}\right), 6.80$ (tt, $J=$ 9.2, $\left.2.4 \mathrm{~Hz}, 1 \mathrm{H}, \mathrm{CH}_{\mathrm{Ar}}\right), 6.92\left(\mathrm{~m}, 2 \mathrm{H}, \mathrm{CH}_{\mathrm{Ar}}\right) ;{ }^{13} \mathrm{C} \mathrm{NMR}\left(100.6 \mathrm{MHz}, \mathrm{CD}_{3} \mathrm{OD}\right) \delta 18.4\left(\mathrm{CH}_{3}\right), 43.6$ $\left(\mathrm{CH}_{2}\right), 50.4(\mathrm{CH}), 103.8\left(\mathrm{t}, J_{\mathrm{C}-\mathrm{F}}=25.6 \mathrm{~Hz}, \mathrm{CH}_{\mathrm{Ar}}\right), 114.0\left(\mathrm{dd}, J_{\mathrm{C}-\mathrm{F}}=18.7,7.0 \mathrm{~Hz}, \mathrm{CH}_{\mathrm{Ar}}\right), 141.9(\mathrm{t}$, $J_{\mathrm{C}-\mathrm{F}}=9.3 \mathrm{~Hz}, \mathrm{C}_{\mathrm{Ar}}$ ), $165.2\left(\mathrm{dd}, J_{\mathrm{C}-\mathrm{F}}=246.8,13.3 \mathrm{~Hz}, \mathrm{C}_{\mathrm{Ar}}\right), 173.3,176.8(\mathrm{C}=\mathrm{O})$. HMRS calcd for $\left[\mathrm{C}_{11} \mathrm{H}_{11} \mathrm{~F}_{2} \mathrm{NO}_{3}+\mathrm{H}\right]: 244.0780$, found: 244.0781 .

(S)- $N$-[4-(7-Benzyl-5,6,7,8-tetrahydroimidazo[2',1':2,3]thiazolo[5,4-c]pyridin-2-yl)phenyl]2-[(3,5-difluorophenyl)acetamido]propanamide (JLK1360). Procedure A. To a mixture of compound 8 (120 mg, $0.49 \mathrm{mmol}$ ) and activated $4 \AA \mathrm{MS}$ in $\mathrm{CH}_{2} \mathrm{Cl}_{2}$ (8 mL) were added BOP (217 mg, $0.49 \mathrm{mmol}$ ), HOBt (66 mg, $0.49 \mathrm{mmol}$ ), and DIPEA (214 $\mu \mathrm{l}, 1.23 \mathrm{mmol}$ ). The resulting mixture was stirred at rt for 20 minutes, and then a solution of amine 5 (89 $\mathrm{mg}, 0.25 \mathrm{mmol}$ ) in $\mathrm{CH}_{2} \mathrm{Cl}_{2}(3 \mathrm{~mL})$ was added via a cannula. After $24 \mathrm{~h}$ of continuous stirring at rt, the reaction was quenched with saturated aqueous $\mathrm{NaHCO}_{3}$. The aqueous layer was extracted with EtOAc, and the combined organic extracts were washed with water and brine, dried, and concentrated. Flash chromatography (6:4 to 7:3 EtOAc-hexane) afforded compound JLK1360 (72 mg, 50\% yield; R $=0.25$ in 4:1 EtOAc-hexane) as a yellow solid. Procedure B. To a mixture of compound 8 (74 $\mathrm{mg}, 0.31 \mathrm{mmol})$ and activated $4 \AA \mathrm{MS}$ in $\mathrm{CH}_{2} \mathrm{Cl}_{2}(4 \mathrm{~mL})$ were added BOPCl (105 mg, 0.42 mmol), HOBt (56 mg, $0.42 \mathrm{mmol}$ ), and DIPEA (192 $\mu \mathrm{l}, 1.10 \mathrm{mmol})$. The resulting mixture was stirred at rt for 20 minutes, and then a solution of amine 5 (100 mg, $0.28 \mathrm{mmol})$ in $\mathrm{CH}_{2} \mathrm{Cl}_{2}$ (3 $\mathrm{mL}$ ) was slowly transferred via a cannula. After $48 \mathrm{~h}$ of continuous stirring at rt, the reaction was quenched with saturated aqueous $\mathrm{NaHCO}_{3}$ and filtered through Celite ${ }^{\circledR}$. The aqueous layer was extracted with EtOAc and the combined organic extracts were washed with water and brine, dried and concentrated. Flash chromatography $\left(\mathrm{CH}_{2} \mathrm{Cl}_{2}\right.$ to $\left.98: 2 \mathrm{CH}_{2} \mathrm{Cl}_{2}-\mathrm{MeOH}\right)$ afforded $33 \mathrm{mg}$ of amine 5 and compound JLK1360 (51 mg, 47\% yield based on consumed 5) as a yellow solid: mp 205-207 ${ }^{\circ} \mathrm{C} ;{ }^{1} \mathrm{H}$ NMR (400 MHz, $\left.\mathrm{CDCl}_{3}\right) \delta 1.43$ (d, $\left.J=7.2 \mathrm{~Hz}, 3 \mathrm{H}, \mathrm{CH}_{3}\right), 2.74$ (t, $J=5.6 \mathrm{~Hz}$, $2 \mathrm{H}, \mathrm{NCH}_{2} \mathrm{CH}_{2}$ ), 2.97 (t, $J=5.6 \mathrm{~Hz}, 2 \mathrm{H}, \mathrm{NCH}_{2} \mathrm{CH}_{2}$ ), 3.54 (s, $2 \mathrm{H}, \mathrm{CH}_{2} \mathrm{CO}$ ), 3.57 (s, $2 \mathrm{H}, \mathrm{NCH}_{2}$ ), 3.76 (s, 2H, $\mathrm{CH}_{2} \mathrm{Ar}$ ), 4.71 (qd, $\left.J=7.2 \mathrm{~Hz}, 1 \mathrm{H}, \mathrm{CH}\right), 6.69$ (t, $J=9.2 \mathrm{~Hz}, 1 \mathrm{H}, \mathrm{CH}_{\mathrm{Ar}}$ ), $6.78(\mathrm{~d}, J=$ $\left.9.2 \mathrm{~Hz}, 1 \mathrm{H}, \mathrm{CH}_{\mathrm{Ar}}\right), 6.79$ (d, $J=9.2 \mathrm{~Hz}, 1 \mathrm{H}, \mathrm{CH}_{\mathrm{Ar}}$ ), 6.97 (br s, 1H, NH), 7.26-7.36 (m, 5H, $\mathrm{CH}_{\mathrm{Ar}}$ ), $7.45\left(\mathrm{dm}, J=7.2 \mathrm{~Hz}, 2 \mathrm{H}, \mathrm{CH}_{\mathrm{Ar}}\right.$ ), $7.46\left(\mathrm{~s}, 1 \mathrm{H}, \mathrm{CH}_{\mathrm{Ar}}\right.$ ), $7.69\left(\mathrm{dm}, J=7.2 \mathrm{~Hz}, 2 \mathrm{H}, \mathrm{CH}_{\mathrm{Ar}}\right), 8.80$ (br s, $1 \mathrm{H}, \mathrm{NH}) ;{ }^{13} \mathrm{C}$ NMR $\left(100.6 \mathrm{MHz}, \mathrm{CDCl}_{3}\right) \delta 17.9\left(\mathrm{CH}_{3}\right), 22.9\left(\mathrm{NCH}_{2} \mathrm{CH}_{2}\right), 42.7\left(\mathrm{CH}_{2} \mathrm{CO}\right), 48.7$ $\left(\mathrm{NCH}_{2} \mathrm{CH}_{2}\right), 49.8(\mathrm{CH}), 50.4\left(\mathrm{NCH}_{2}\right), 61.3\left(\mathrm{CH}_{2} \mathrm{Ar}\right), 102.9\left(\mathrm{t}, J_{\mathrm{C}-\mathrm{F}}=24.9 \mathrm{~Hz}, \mathrm{CH}_{\mathrm{Ar}}\right), 105.5(\mathrm{CH})$, $112.2\left(\mathrm{dd}, J_{\mathrm{C}-\mathrm{F}}=18.7,7.0 \mathrm{~Hz}, \mathrm{CH}_{\mathrm{Ar}}\right), 119.1\left(\mathrm{C}_{\mathrm{Ar}}\right), 120.2\left(\mathrm{CH}_{\mathrm{Ar}}\right), 124.5\left(\mathrm{C}_{\mathrm{Ar}}\right), 125.6,127.5$, 128.5, $129.0\left(\mathrm{CH}_{\mathrm{Ar}}\right), 130.6,136.6,137.6\left(\mathrm{C}_{\mathrm{Ar}}\right), 138.0\left(\mathrm{t}, J_{\mathrm{C}-\mathrm{F}}=10.1 \mathrm{~Hz}, \mathrm{C}_{\mathrm{Ar}}\right), 146.1,148.4\left(\mathrm{C}_{\mathrm{Ar}}\right)$, 163.1 (dd, $\left.J_{\mathrm{C}-\mathrm{F}}=249.2,13.3 \mathrm{~Hz}, \mathrm{C}_{\mathrm{Ar}}\right), 170.1(\mathrm{C}=\mathrm{O}), 170.2(\mathrm{C}=\mathrm{O})$; $[\alpha]_{\mathrm{D}}^{22}-7.6(c 0.11, \mathrm{MeOH})$. HMRS calcd for $\left[\mathrm{C}_{32} \mathrm{H}_{29} \mathrm{~F}_{2} \mathrm{~N}_{5} \mathrm{O}_{2} \mathrm{~S}+\mathrm{H}\right]$ : 586.2083, found: 586.2071. 


\section{Acknowledgements}

Financial support from the Ministry of Science and Technology (Spain)-FEDER (project CTQ2006-02390/BQU) and the DURSI, Generalitat de Catalunya (Grant 2005-SGR-0603) is gratefully acknowledged. We also acknowledge for COST Short-Term Scientific Missions for N. L. and F. S.

\section{References}

1. Patané, S.; Pietrancosta, N.; Hassani, H.; Leroux, V.; Maigret, B.; Kraus, J.-L.; Dono, R.; Maina, F. Biochem. Biophys. Res. Commun. 2008, 375, 184.

2. For the synthesis of the 5-Boc derivative, see: Haginoya, N.; Komoriya, S.; Osanai, K.; Yoshino, T.; Nagata, T.; Nagamochi, M.; Muto, R.; Yamaguchi, M.; Nagahara, T.; Kanno, H. Heterocycles 2004, 63, 1555.

3. For the use of this procedure for the construction of 2-aryl substituted imidazo[2,1- $b$ ] thiazoles, see: (a) Munakata, M. US Patent 4, 244, 952, 1981; Chem. Abstr. 1977, 87, 68236h. (b) Mase, T.; Arima, H.; Tomioka, K.; Yamada, T.; Murase, K. J. Med. Chem. 1986, 29, 386. (c) Barchéchath, S. D.; Tawatao, R. I.; Corr, M.; Carson, D. A.; Cottam, H. B. J. Med. Chem. 2005, 48, 6409. (d) Pietrancosta, N.; Moumen, A.; Dono, R.; Lingor, P.; Planchamp, V.; Lamballe, F.; Bähr, M.; Kraus, J.-L.; Maina, F. J. Med. Chem. 2006, 49, 3645.

4. For the synthesis of 2-imino-2,3,4,5,6,7-hexahydrobenzothiazole derivatives bearing a phenacyl substituent on the ring nitrogen, see: (a) Zhu, X.; Yu, Q.-S.; Cutler, R. G.; Culmsee, C. W.; Holloway, H. W.; Lahiri, D. K.; Mattson, M. P.; Greig, N. H. J. Med. Chem. 2002, 45, 5090. (b) Pietrancosta, N.; Maina, F.; Dono, R.; Moumen, A.; Garino, C.; Laras, Y.; Burlet, S.; Quéléver, G.; Kraus, J.-L. Bioorg. Med. Chem. Lett. 2005, 15, 1561.

5. For a previous synthesis of $\mathbf{8}$ by TFA treatment of the corresponding tert-butyl ester, see: Pietrancosta, N.; Quéléver, G.; Laras, Y.; Garino, C.; Burlet, S.; Kraus, J. L. Aust. J. Chem. 2005, 58, 585.

6. (a) Ramamoorthy, P. S.; Gervay, J. J. Org. Chem. 1997, 62, 7801. (b) Dormoy, J.-R.; Castro, B. In Encyclopedia of Reagents for Organic Synthesis; Paquette, L. A. Ed.; John Wiley \& Sons: New York, 1995; Vol. 1, pp. 301-304. (c) Zhang, A.; Schlüter, A. D. Chem. Asian J. 2007, 2, 1540.

7. Li, P.; Xu, J.-C. Tetrahedron 2000, 56, 8119.

8. Rees, D. C.; Hamilton, N. M. In Encyclopedia of Reagents for Organic Synthesis; Paquette, L. A. Ed.; John Wiley \& Sons: New York, 1995; Vol. 1, pp. 543-544. 